\title{
The methodological approach to environmental risk assessment from man-made emergencies at chemically hazardous sites
}

\author{
Pavel V. Yemelin ${ }^{1^{*}}$, Sergey S. Kudryavtsev ${ }^{2^{+*}}$, Natalya K. Yemelina ${ }^{3}$ \\ ${ }^{1}$ Department of Merchandising and Certification, Karaganda Economical University, Karaganda, Kazakhstan \\ ${ }^{2}$ Department of Chemistry and Chemical Technologies, Karaganda Technical University, Karaganda, Kazakhstan \\ ${ }^{3}$ Department of Statistics and Data Analysis, National Research University Higher School of Economics, Moscow, Russian Federation \\ "These authors contributed equally to this work.
}

\section{ABSTRACT}

The purpose of the article is to present a methodological approach to assessing the state of the risk to the environment from man-made emergencies at chemically hazardous sites. The methodological approach is based on an expert method, the use of which allowed the authors to create the structure of the risk management system and, determine the plurality of criteria parameters while taking into account their weight in the formation of the environmental consequences of chemical accidents. Criteria parameters were grouped into clusters of physicochemical and toxicological properties of hazardous chemicals used at a chemical plant, equipment operating at an enterprise, personnel, and environmental vulnerability. The research became the basis of the Guidelines for Assessing the Environmental Risk from Emergencies at Chemically Hazardous Sites. As an example, the article presents the calculation of the level of environmental risk for a terrestrial site from the metallurgical enterprise LLP Kazakhmys Smelting, in Zhezkazgan town, Republic of Kazakhstan. The developed methodology is the basis of the Information and Analytical System for Assessing the Environmental Consequences of Man-made Emergencies, which allows users to facilitate the implementation of a set of measures aimed to forecast and reduce the impact of emergency's damaging factors on the environment.

Keywords: Chemical safety, Environmental risk, Environmental risk assessment, Environmental safety, Hazardous chemicals

\section{Introduction}

The main purpose of risk assessment is its forecasting [1, 2]. Each chemical accident has certain features, and therefore environmental risk assessment can and should be carried out in different ways. Currently, there is no unified forecasting mechanism, and environmental risk assessment uses tools that are viewed by risk assessment experts as necessary. Environmental risk is the likelihood of adverse changes in the state of the environment and (or) natural objects due to the influence of certain factors [3]. Against this background, it is possible to consider chemical accidents from the standpoint of risk assessment.

Hazardous chemicals are chemicals, the direct or indirect impact of which on a human being can cause acute and chronic diseases or death [4]. Especially dangerous consequences are typical for emergencies at enterprises that use hazardous chemicals, since they have a detrimental effect not only on people, but also on the environment, causing gas pollution, contamination of land and water.

The environmental disaster that occurred in August 2014 in the state of Sonora, Mexico is widely known [5]. As a result of the emergency, about 40 thousand cubic meters of contaminated wastewater containing sulfuric acid from the Buenavista copper mine in Cananea fell into the river system of the Bacanuchi river tributary of the Sonora river [6]. This disaster caused the death of a huge number of aquatic organisms. Also, the accident had a tangible impact on the coastal ecosystem.

Chemically hazardous sites are sites where hazardous chemicals are stored, processed, used or transported, in case of an accident or destruction of which, death or chemical contamination of people, agricultural animals and plants, as well as chemical contamination of the natural environment may occur [4]. A significant part of non-chemical industries, where hazardous chemicals are used in technological processes and chemical transformations take place
This is an Open Access article distributed under the terms of the Creative Commons Attribution Non-Commercial License (http://creativecommons.org/licenses/by-nc/3.0/) which permits unrestricted non-commercial use, distribution, and reproduction in any medium, provided the original work is properly cited.

Copyright (C) 2021 Korean Society of Environmental Engineers
Received July 09, 2020 Accepted August 30, 2020

${ }^{\dagger}$ Corresponding author

Email: sk74_07@mail.ru

Tel: $+7(7212) 564422$

ORCID: 0000-0002-7232-6304 
that can lead to an emergency, also refers to chemically hazardous sites. The danger of chemically hazardous sites for humans and the environment can also occur with the normal regulated functioning, since, due to their nature and properties, these substances harm human health and the environment.

The analysis of the literature highlighted gaps for further study. Over the past decade, several articles have been published, on the physicochemical and ecotoxicological properties of hazardous chemicals [7-11], environmental vulnerability [12-18], and causes of accidents at chemical plants and factors affecting accident rate [19-21]. However, using them in their original form for the purpose of this research will not enable an objective and comprehensive analysis of the risk to the environment, since one of its aspects or another will remain unexplored.

Assessing the risks associated with handling chemicals is a vast field of study that is being intensively researched. As a rule, the developed models can be divided into two groups: one is associated with long-term consequences, and the other with acute consequences [12].

The long-term effects models cover the transport and fate of chemicals. Often these are persistent organic pollutants and heavy metals, both after an instantaneous and diffuse release of chemicals. Models include both human health risk assessments and long-term environmental risk assessments that take into account the persistence, degradation and bioaccumulation of chemicals [22].

Models associated with acute consequences are often developed to facilitate land use planning, as well as the classification and/or ranking of chemicals and industrial sites to facilitate the organization of preventative measures. These models, as a rule, are based on the internal properties of chemicals, and not on the properties and characteristics of the accident site [23].

The mathematical model developed by the authors [9] is aimed at determining the danger of pollution of the marine (aquatic) environment of chemicals with wildly different toxicity mechanisms, environmental fate, and physicochemical properties.

In Australia, under the leadership of the National Council for the Protection of the Environment and Heritage (EPHC), the National Environmental Chemicals Management Rules (NChEM) have been developed [7]. In addition to the results of the author's own research, this document contains the experience of the states of the European Union, UK, USA, and Canada. It consists of four interrelated areas of activity, covering environmental risk assessment, environmental control, information feedback and the prioritization of actions. In this methodology, a scenario is considered when the emissions of chemicals are not accidental, but technological in nature and occur through treatment plants.

The Guidelines on Information Requirements and Chemical Safety Assessment developed by the European Chemical Agency [9] and adopted in the EU countries describes a methodology for assessing the environmental impact of chemicals. This technique is used to quantify risk and allows users to determine the predicted environmental concentration (PEC) and the predicted non-exposure concentration (PNEC) for organisms.

Multifactorial models have been used in risk assessment methods based on the expert method [24] and in creating an information-analytical risk forecasting system [25]. This methodological approach seemed appropriate to assess the risk of the environ- mental impact taking into account a number of features of current research, among which there is a comprehensive assessment of all the factors that determine the environmental risk from chemically hazardous sites. Moreover, this will make it possible to analyze the environmental risk of accidental release of hazardous chemicals comprehensively from the point of view of chemical, environmental, and industrial safety.

The Methodology for Environmental Risk Assessment from Emergencies at Chemically Hazardous Sites is based on the analysis of statistical data on accidents that have occurred, theoretical researches on the reliability of technological processes, modeling risk situations, and conducting risk assessments of various accidents at enterprises.

Developed on the basis of this methodology, the Information and Analytical System for Assessing the Environmental Consequences of Man-made Emergencies allows the ecological departments of enterprises to automate the process of the environmental risk assessment from emergencies at chemically hazardous sites.

Application of the results will allow governments, companies, and the industrial sector as a whole to plan and implement measures to reduce risks and mitigate the consequences of possible accidents and catastrophes that may occur at hazardous chemical sites.

\section{Material and Methods}

The assessment of the environmental risk from man-made emergencies at chemically hazardous sites was carried out by the authors in three stages:

(1) Preliminary research;

(2) Collection and formation of a database of initial information, and

(3) Environmental risk level assessment.

The flow chart of the process of assessing the environmental risk from man-made emergencies at chemically hazardous sites is shown in Fig. 1.

The purpose of the preliminary stage of the study was to determine the criteria parameters for two risk components: the index of the environmental hazard of a chemically hazardous site and the index of the environmental vulnerability from a chemically hazardous site.

The index of the environmental hazard of a chemically hazardous site is quantitatively measured according to the established criteria, the possibility that an accident will occur.

The environmental vulnerability index characterizes the degree of environmental vulnerability when exposed to a damaging factor as a result of man-made emergencies.

For the first stage, the method of expert assessments was applied. To date, there is in the Republic of Kazakhstan the registration of accidents at enterprises that have caused exclusively moderate injuries, severe injuries and deaths. Such emergencies are not always associated with harm to the environment, while accidents that have caused, or are likely to cause harm to the environment, may not be recorded in the statistics of the authorized organizations. This fact causes significant difficulties in determining the posterior risk of accidents at chemically hazardous sites. In this regard, the 


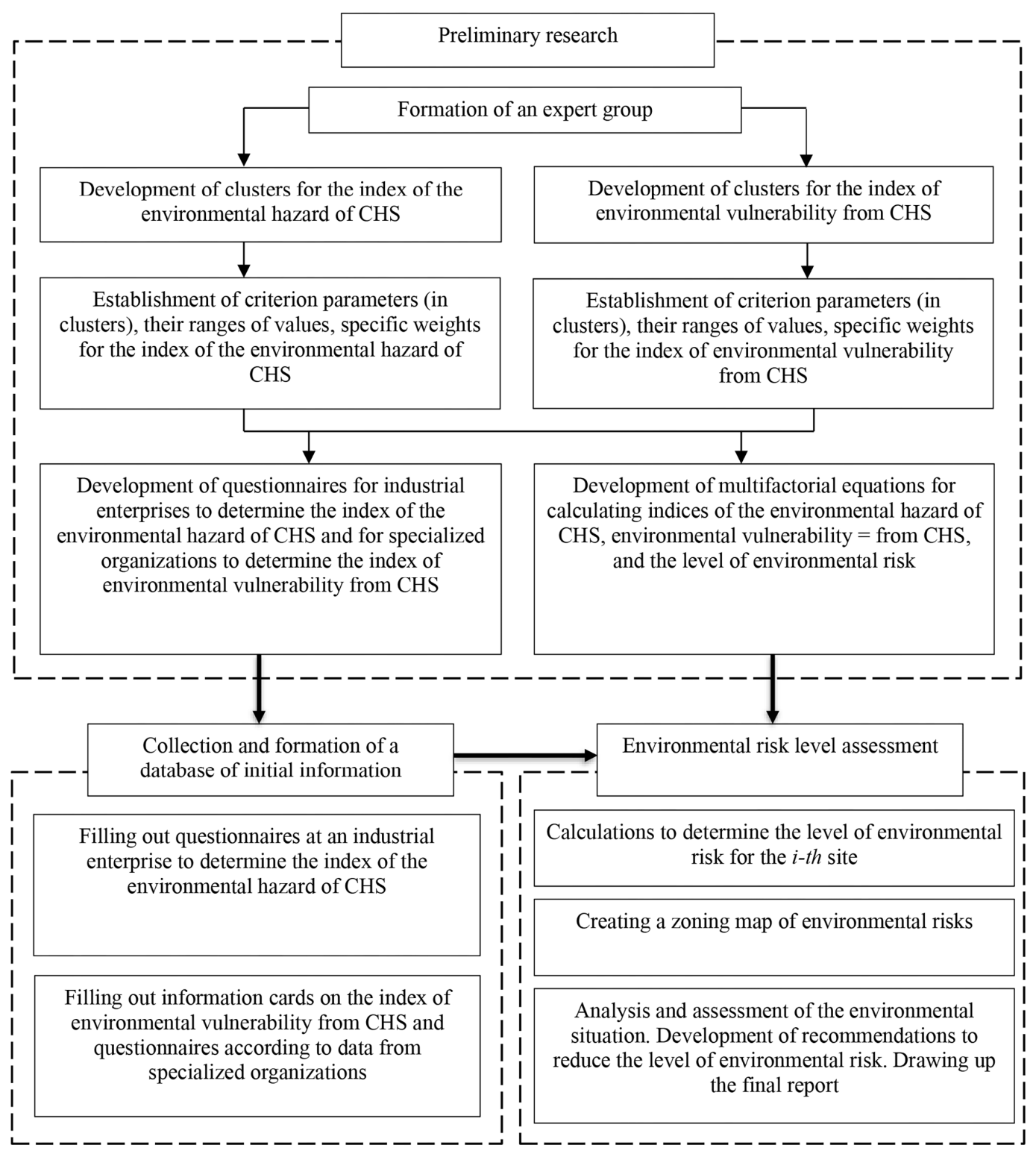

Fig. 1. Flow chart of the process for assessing the environmental risk from man-made emergencies at $\mathrm{CHS}$.

absence of an objective accident database, along with many disparate factors that determine the frequency and severity of environmental consequences and environmental vulnerability, makes the use of the expert assessment method relevant to our methodology.

A. Anderson et al. [26, 27] showed a high reliability of the results of applying expert methods in assessing environmental risk.

When selecting experts, a basic list of candidates was formed, and then a preliminary and final one. The formation of the basic list of candidates for the expert group was carried out according to the classical method of "snowball."

27 highly qualified specialists in the field of industrial, chemical, and environmental safety with an average experience of 14,5 years were included in the final list of experts:

- employees of the Committee for Emergency Management of the Ministry of Internal Affairs of the Republic of Kazakhstan - 5 people;

- employees of the Industrial Safety Committee of the Ministry of Industry and Infrastructural Development of the Republic of Kazakhstan - 4 people;

- employees of the Industrial Safety Department in the Karaganda region - 3 people;

- employees of the Department of Ecology in the Karaganda region - 4 people;

- employees of organizations involved in environmental impact assessment - 5 people;

- employees of chemically hazardous sites responsible for the safety of their operation - 6 people.

The high level of competence of these employees in the issues 
we are studying along with their optimal number in the sample allowed us to obtain reliable results.

The method of expert evaluations is based on gathering opinions on the posed problem, the analysis, and processing of received judgments and the generalization of the survey results. Extensive knowledge of the subject of research and the accumulated experience of the expert allows us to compensate for the lack of information or the inability to use it to model the possible consequences of technological accidents at chemically hazardous sites.

An expert survey included several tasks. Based on the opinions of experts, the following were determined (Tables S1-S10):

- the main directions (clusters) of influence on indexes of environmental hazard of a chemically hazardous site and environmental vulnerability;

- weight coefficients for each cluster when determining definite index (hazard, vulnerability);

- criteria parameters that describe each cluster as fully as possible; and

- the degree and significance of each criteria parameter for assessing the environmental consequences of man-made emergencies.

In the developed methodological approach, the research considered a peacetime emergency that occurred according to the most pessimistic scenario [28]. This scenario implied the destruction of the largest volume of hazardous substance - filled reservoir, leading to the complete leakage and/release of this substance [28-30], which would have the most harmful effect on the environment.

Weighting coefficients for each cluster and the significance of all criteria parameters was determined using a linguistic scale (Table 1). The scale is necessary to unify the qualitative characteristics and quantitative scores. According to a qualitative description of the values of the criteria parameter, their quantitative mark is determined on a scale from 0 to 9 . The absence of the influence of the value of the criteria parameter meets the " 0 " mark. The maximum influence of the value of the descriptor meets the " 9 " mark.

Taking into account the obtained results of expert assessment, the weight coefficients were calculated by the equation:

$$
w_{j}=\frac{\bar{g}_{j}}{\sum_{j=1}^{n} \bar{g}_{j}}
$$

where $\bar{g}_{j}$ is the arithmetic mean of scores from experts on the $j$-th parameter.

The weight coefficients of the criteria parameters (Tables S1-S10), obtained using the expert method, were then used by the authors to develop multifactorial equations for calculating the indices of the environmental hazard of CHS's and environmental vulnerability of CHS's, as well as the level of environmental risk, which are presented in Sections 2.3- 2.5.

In addition, based on the list of criteria parameters, questionnaires were formed for industrial enterprises to determine the index of the environmental hazard of CHS. Also, from the appropriate descriptors, questionnaires were compiled, which were used by specialized organizations to determine the index of environmental vulnerability from CHS.

The second stage of assessing the environmental risk of a chemically hazardous site (Fig. 1) includes the collection and formation of a database of initial information. On the basis of a questionnaire survey carried out at industrial enterprises, the index of the environmental hazard of CHS is determined. Filling out questionnaires according to the data of specialized organizations and information cards allows us to determine the index of environmental vulnerability from CHS.

The information obtained as a result of the questionnaire survey, as well as the developed equations for assessing the indices of the environmental hazard of CHS and environmental vulnerability from CHS, allow researchers to assess the risk level of environmental consequences as a result of emergencies at chemically hazardous sites for a definite $i$-th area of the environment (Fig. 1). Calculation for a set of $i$-th areas of the environment around the enterprise allows us to create maps of environmental risks' zoning. These maps are the basis for analyzing and assessing the environmental situation at an industrial enterprise's CHS, developing measures and recommendations aimed at reducing the level of environmental risk.

The methodology for assessing the environmental hazard of a

Table 1. Scale for Assessing the Significance (degree of influence) of the Criteria Parameter

\begin{tabular}{|c|c|c|}
\hline \multirow{2}{*}{$\begin{array}{l}\text { Degree of } \\
\text { influence }\end{array}$} & \multicolumn{2}{|l|}{ Mark } \\
\hline & Linguistic & Score \\
\hline \multirow[t]{2}{*}{ Absents } & No degree of impact on the environmental hazard of a chemically hazardous site/environmental vulnerability & 0 \\
\hline & Very low degree of impact on the environmental hazard of a chemically hazardous site/environmental vulnerability & 1 \\
\hline \multirow[t]{3}{*}{ Weak } & Low degree of impact on the environmental hazard of a chemically hazardous site/environmental vulnerability & 2 \\
\hline & Insignificant degree of impact on the environmental hazard of a chemically hazardous site/environmental vulnerability & 3 \\
\hline & Lower than medium degree of impact on the environmental hazard of a chemically hazardous site/environmental vulnerability & 4 \\
\hline \multirow[t]{3}{*}{ Medium } & Medium degree of impact on the environmental hazard of a chemically hazardous site/environmental vulnerability & 5 \\
\hline & Above medium degree of impact on the environmental hazard of a chemically hazardous site/environmental vulnerability & 6 \\
\hline & Significant degree of impact on the environmental hazard of a chemically hazardous site/environmental vulnerability & 7 \\
\hline \multirow[t]{2}{*}{ Strong } & Strong degree of impact on the environmental hazard of a chemically hazardous site/environmental vulnerability & 8 \\
\hline & Very strong degree of impact on the environmental hazard of a chemically hazardous site/environmental vulnerability & 9 \\
\hline
\end{tabular}


CHS, environmental vulnerability from CHS and environmental risk level is set out below.

\subsection{Hazard Index of a Chemically Hazardous Site}

The hazard index of a chemically hazardous site for the environment is determined by the total influence of factors combined into three clusters:

- the cluster of criteria parameters for physicochemical, toxicological properties and the number of hazardous chemicals;

- the cluster of criteria parameters for equipment used at an industrial enterprise; and

- the cluster of criteria parameters for personnel working at an industrial enterprise.

Table 2 shows the distribution of the weights of these clusters.

\subsubsection{Cluster of descriptors for physicochemical, toxicological} properties and amount of hazardous chemicals

The severity of environmental consequences, primarily, is associated with chemicals located at the CHS, their quantity, physicochemical and toxicological properties. When creating the methodology, we took the physicochemical and ecotoxicological properties of hazardous chemicals, considered in the works of Häkkinen et al. [8, 9], who studied such a kind of risk in cases of accidents in maritime and land transport, simplifying the characteristics of hazardous chemicals given in EpiSuite [10] to three quantitative expressions.

Most of the descriptors had five quantitative expressions for more convenient and objective operation in the proposed methodology. This allowed the researchers to make the assessment of the hazard of CHS more comprehensively. To determine the values of physicochemical and ecotoxicological properties of hazardous chemicals, data from the sites of the European Chemical Agency (ECHA) (https://www.echa.europa.eu/) and National Institutes of Health, USA (https://pubchem.ncbi.nlm.nih.gov/) were utilized. In the absence of factual data about the half-life (biodegradation) of some hazardous chemicals, the results of an expert assessment $\mathrm{BIOWIN}_{3}$ (US Environmental Protection Agency) were used.

Also, in the proposed methodology, a descriptor of the volume of hazardous chemicals in circulation at CHS is additionally used. Its quantitative expression is determined by Part II of Annex I of Directive 2012/18/EU of the European Parliament and of the Council of 4 July 2012 On the Control of Major-accident Hazards Involving Dangerous Substances (Seveso III) (2012/18/EU) [31]. This directive also distinguishes two hazard levels of enterprises depending on the number of chemicals available on them and their properties: lower-tier establishment and upper-tier establishment.
As the analysis of published data [8, 9] showed, the criteria parameters describing the environmental risks of accidents with the spill of hazardous chemicals into the soil have some differences from those for the aquatic environment. In this regard, in the proposed methodology, these cases will be considered separately. Tables S1 and S2 show descriptors that determine the relationship between the properties of hazardous chemicals and their effect on environmental sites in the aquatic environment and soil, respectively.

When determining the value of acute and chronic toxicity, the minimum value for one of the given trophic levels (fish, invertebrates or algae) should be selected.

In the absence of values determined for the soil, the values determined for the aquatic medium are used.

The choice of these criteria parameters is determined by the completeness of the description of the following properties of a hazardous chemical that affect the environmental hazard of a chemically hazardous site.

\subsubsection{Cluster of criteria parameters of equipment used at an in- dustrial enterprise}

Table S3 presents the descriptors included in the cluster of equipment used at the industrial enterprise, their quantitative expressions, and weight coefficients.

\subsubsection{Cluster of descriptors for personnel working at an industrial enterprise}

Table S4 presents the descriptors included in the cluster of personnel working at an industrial enterprise, their quantitative expressions, and weight coefficients.

\subsection{Index of Environmental Vulnerability from a Chemically Hazardous Site}

Anderson [12] considers among environmental vulnerability descriptors the distance to a water site, the depth of groundwater, the slope of the earth's surface and ground characteristics. It does not matter whether the environmental site is on land (soil) or an aquatic site, in determining the index of environmental vulnerability. Table S5 shows descriptors for the index of environmental vulnerability from a chemically hazardous site to the territory and the water area, and as can be seen, they have noticeable differences.

We see the use of soil type as criteria parameter not entirely justified. The fact is that the soil is not always homogeneous; often the thickness of the soil is formed by layers having different permeability. In this regard, it will be more appropriate to use the degree of water permeability of the soil as a descriptor, which

Table 2. Clusters Describing the Hazard Index of a Chemically Hazardous Site for the Environment

\begin{tabular}{lc}
\hline Cluster name & $\begin{array}{c}\text { The total value of the weight } \\
\text { coefficient for cluster }\end{array}$ \\
\hline Cluster of descriptors for physicochemical, toxicological properties and amount of hazardous chemicals & 0,6 \\
Cluster of descriptors for equipment used at an industrial enterprise & 0,23 \\
Cluster of descriptors for personnel working at an industrial enterprise & 0,17 \\
Total weight & 1
\end{tabular}


can be judged by the filtration coefficient.

Appropriate information maps are used for the collection and analysis of source data, such as surface topography, hydrogeological parameters, ranges and ratings for soil. In the absence of information on hydrogeology and soil characteristics, it is necessary to use the data obtained for the sites closest to the terrestrial or aquatic research area. Combining maps of various physiographic attributes allows the creation of vulnerability maps of the researched area.

\subsubsection{Descriptors for assessing the environmental vulnerability from a chemically hazardous site}

The Distance to the Researched Land Plot (Soil), Site/Water Protection Zone (Well, Lake or Watercourse). In its integral form, this index reflects the subordination of flat, transit, and accumulative landscapes that determine the paths of migration and accumulation of pollutants. Thus, the closer the assessed point is to the considered land plot or watercourse, the higher the probability of contaminants entering the plot or surface water and transporting them over significant distances. It is also necessary to take into account that at the boundaries of the separation of media (land - water), geochemical processes occur most actively, as a result of which mechanical influences can create significant disturbances in their dynamics and, geochemical barriers and actively destabilize the dynamics of natural complexes. Table S6 presents the quantitative expression of this parameter and its score scale.

The distance to the researched land or aquatic site is calculated according to the equation given in the Digest of Methodologies for Forecasting Possible Accidents, Catastrophes, and Natural Disasters in RFEM [26].

If there is some slope towards the researched site, then the spill diameter is adjusted by taking into account the angle of slope. If the distance to the research site / (well, lake or watercourse) is longer than the calculated value of the possible distribution of a hazardous chemical or more than the values of Table S6, then we assume that this hazardous chemical cannot cause environmental harm to this site. Accordingly, the environmental vulnerability index from CHS for this i-th site is equal to zero. The degree of environmental vulnerability from a chemically hazardous site for the i-th land plot (soil), site or aquatic medium is absent.

Depth to Groundwater-Surface. Aquifers in the researched territories can be located at depths from 0.2 to $60 \mathrm{~m}$ and deeper. As a result, pollutants can be transported over long distances. As for mechanical influences, the higher the groundwater level, the more actively they will affect its hydrodynamic indicators (increases, or decreases in levels).

If the depth to the surface of groundwater is higher than the calculated value of the possible distribution of a hazardous chemical or more than the values of Table S7, then it is accepted that this hazardous chemical cannot cause environmental damage to this site through groundwater. Accordingly, the index of environmental vulnerability from CHS for this site is taken equal to zero. The degree of environmental vulnerability from a chemically hazardous site for the i-th aquatic medium is absent.

Slope of the Earth's Surface / (Groundwater and Flow Direction). This parameter generally determines the migration ability of pollutants. The more actively they spread throughout the land complex, the more significant will be the destabilizing effect on all its components. With an increase in the slope, various slope processes will also become more active, which can significantly affect the results of environmental impact (Table S8).

Degree of Water Permeability of the Soil. According to the Interstate Standard 25100-95 Soils. Classification [32], soils are divided into five categories by the degree of permeability: non-water permeable; poorly water permeable; water permeable; highly water permeable; and, very highly water permeable. With an increase in the value of this descriptor, the risk of chemical pollution of groundwater that has a direct connection with a water site (wells, lakes or watercourse) increases (Table S9). The higher this descriptor's value, the more active is the transport of pollutants. In this case, watercourses, on the one hand, act as migration corridors, and on the other, as potential sites of chemical contamination. At the same time, there is an opposite effect when considering the influence of this criteria parameter for a land plot (soil), or a site located on the earth's surface.

The Purpose of the Land Plot (Soil), Site/Reservoir. Taking into account the current situation and land use development plans, the distribution and, purpose of the researched territories is presented in the following form: nature reserve; basic farmland; unused land; and other types. For water sites, the purpose of classification is presented as drinking, water industrial, welfare, natural wetlands, and other types. The vulnerability of a site will accordingly be determined by the scale of protective measures and the degree of its vulnerability [13, 33] (Table S10).

Analysis and generalization of different methods for assessing vulnerability for chemical and mechanical effects have shown the feasibility of creating a generalized integrated matrix for assessing vulnerability using the same descriptors. The idea is that even if it is possible to classify the effects on different classes and subclasses, they remain essentially multifactorial [33].

The described set of matrix parameters is basic. Along with them, it is possible to use additional ones that would reflect the specificity of the characteristics inherent in individual objects of assessment. This approach to the analysis of the assessment is called a two-level approach [33]. Its purpose is to increase the representation of the assessment by introducing a second matrix of parameters (for example, the degree of disturbance of land, the purpose of land, etc.). Using just such an approach allows researchers to cover the entire spectrum of characteristics, but at the same time to divide them into priority ones or those whose contribution is most significant for vulnerability assessment, and less significant, but still having importance and affecting the calculation of the total integral index.

As a result of the research, clusters of descriptors are formed for hazard and vulnerability indexes that describe them; determine their total weight of influence on a particular index; and establish possible ranges of values for each criteria parameter regarding scores, as well as their weights in the cluster in which they are defined.

\subsection{Calculation and Assessment of the Environmental Hazard Index of a Chemically Hazardous Site}

The environmental hazard index of a chemically hazardous site for the $i$-th land plot (soil), site or aquatic media is determined 
by the data on the degree of influence of the set of criteria parameters $\{\mathrm{n}\}$ and the significance of their values:

$$
E H I_{i}=\sum_{k=1}^{3} \sum_{j=1}^{n} w_{k j} \cdot f_{k j}
$$

where $w_{\mathrm{kj}}$ is the weight of the $j$-th criteria parameter, calculated by the equation (1);

$\mathrm{fj}$ is an importance rating of the value of the criteria parameter; and $\mathrm{n}$ is the number of criteria parameters for the $k$-th cluster of the environmental hazard index of a chemically hazardous site.

The environmental hazard index of a chemically hazardous site for the $i$-th land plot (soil), site or aquatic media will be determined as:

$$
\begin{gathered}
E H I_{i}=\left(0.05 \cdot\left(f_{11}+f_{13}+f_{14}\right)+0.04 \cdot f_{12}+\right. \\
\left.0.06 \cdot\left(f_{15}+f_{17}\right)+0.12 \cdot f_{16}+0.17 \cdot f_{18}\right)+ \\
\left(0.037 \cdot f_{21}++0.035 \cdot f_{22}+0.025 \cdot f_{23}+\right. \\
\left.0.03 \cdot\left(f_{24}+f_{27}\right)+0.033 \cdot f_{25}+0.04 \cdot f_{26}\right)+ \\
\left(0.07 \cdot f_{31}+0.1 \cdot f_{32}\right)
\end{gathered}
$$

where according to the cluster of criteria parameters for the physicochemical, toxicological and other properties of a hazardous chemical for a land plot (soil), a site:

- $f_{11}$ is an importance rating of volatility (vapor pressure);

- $f_{12}$ is an importance rating of mobility in the soil (density/viscosity ratio);

- $f_{13}$ is an importance rating of mobility (by solubility in water);

- $f_{14}$ is an importance rating of constancy (BIOWIN ${ }_{3}$ half-life);

- $f_{15}$ is an importance rating of bioaccumulation (log $\mathrm{K}_{\mathrm{OW}}$ );

- $f_{16}$ is an importance rating of acute toxicity (LC / EC50);

- $f_{17}$ is an importance rating of chronic toxicity (NOEC);

- $f_{18}$ is an importance rating of the storage volume; where according to the cluster of criteria parameters for physicochemical, toxicological and other properties of a hazardous chemical for the aquatic medium:

- $f_{11}$ is an importance rating of volatility (vapor pressure);

- $f_{12}$ is an importance rating of the density of a hazardous chemical $\mathrm{g} / \mathrm{cm}^{3}$;

- $f_{13}$ is an importance rating of water solubility;

- $f_{14}$ is an importance rating of constancy (BIOWIN $N_{3}$ half-life);

- $f_{15}$ is an importance rating of bioaccumulation (log $\mathrm{K}_{\mathrm{OW}}$ );

- $f_{16}$ is an importance rating of acute toxicity (LC / EC50);

- $f_{17}$ is an importance rating of chronic toxicity (NOEC);

- $f_{18}$ is an importance rating of the storage volume;

for a cluster of criteria parameters for equipment used at an enterprise:

- $f_{21}$ is an importance rating of the length of piping systems transporting hazardous chemicals;

- $f_{22}$ is an importance rating of the wear coefficient of fixed assets;

- $f_{23}$ is an importance rating of the replacement coefficient of fixed assets;
- $f_{24}$ is an importance rating of the capacity of pumps (compressors); - $f_{25}$ is an importance rating of the number of storage tanks for hazardous chemicals at the site;

- $f_{26}$ is an importance rating of the presence of automatic shut-off valves;

- $f_{27}$ is an importance rating of the presence of localization tools; where according to the cluster of criteria parameters for personnel working at the industrial enterprise:

- $\mathrm{f}_{31}$ is an importance of the number of employees at CHS who were trained in the course "Industrial Safety at Hazardous Industrial Sites" or another advanced training course (for the reporting period), \%;

- $\mathrm{f}_{32}$ is an importance of organizational activities aimed at the trouble-free operation of manufacture.

The weight coefficients for equation (3) are taken from Tables S1-S4.00

The calculated values of the environmental hazard indexes of a chemically hazardous site according to equation (3) meet to a certain degree of environmental hazard of a chemically hazardous site, as presented in Table 3. The scale has five ranges of values with the corresponding degrees of environmental hazard of a chemically hazardous site: negligible; moderate; significant; or, high. The accident hazard index varies from 0 to 5 . If the index is zero, there is no accident hazard, and if the index is near 5 , the accident hazard is high.

Table 3. Scale for Determining the Degree of Environmental Hazard of a Chemically Hazardous Site

\begin{tabular}{cc}
\hline $\begin{array}{c}\text { Intervals of the environmental } \\
\text { hazard index of a chemically } \\
\text { hazardous site }\end{array}$ & $\begin{array}{c}\text { Degree of environmental } \\
\text { hazard of a chemically } \\
\text { hazardous site }\end{array}$ \\
\hline $0<E H I_{i} \leq 2$ & Negligible \\
$2<E H I_{i} \leq 3$ & Moderate \\
$3<E H I_{i} \leq 4$ & Significant \\
$4<E H I_{i} \leq 5$ & High \\
\hline
\end{tabular}

\subsection{Calculation and Assessment of an Index of Environmental Vulnerability from a Chemically Hazardous Site}

The index of environmental vulnerability from a chemically hazardous site for the $i$-th land plot (soil), site or aquatic medium is determined based on data on the degree of influence of many criteria parameters $\{\mathrm{m}\}$ and the significance of their values:

$$
E V I_{i}=\sum_{j=1}^{m} a_{j} \cdot y_{j}
$$

where $a_{j}$ is the weight of the $j$-th criteria parameter of vulnerability, calculated by the Eq. (1);

$y_{j}$ is the importance rating of the criteria parameter of vulnerability; and $\mathrm{m}$ is the number of criteria parameters forming a cluster for assessing the index of environmental vulnerability from a chemically hazardous site (for a land plot (soil), a site, and an aquatic medium).

The index of environmental vulnerability from a chemically haz- 
ardous site for the $i$-th land plot (soil), the site is determined as:

$$
E V I_{i}=0.4 \cdot y_{1}+0.25 \cdot y_{2}+0.15 \cdot y_{3}+0.2 \cdot y_{4}
$$

where $y_{1}$ is importance rating of the distance to the assessed land (soil), site, m;

$y_{2}$ is an importance rating of the slope of the earth's surface;

$y_{3}$ is an importance rating of the degree of water permeability of the soil, $\mathrm{m} /$ day; and

$y_{4}$ is an importance rating of the purpose of the land (soil), site.

The index of environmental vulnerability from a chemically hazardous site for the $i$-th site of the aquatic medium is defined as:

$$
E V I_{i}=0.4 \cdot y_{1}+0.15 \cdot y_{2}+0.1 \cdot y_{3}+0.15 \cdot y_{4}+0.2 \cdot y_{5},(6)
$$

where $y_{1}$ is an importance rating of the distance to the researched well, lake or watercourse;

$y_{2}$ is an importance rating of depth to the surface of groundwater; $y_{3}$ is an importance rating of the slope of the surface of groundwater and the direction of flow;

$y_{4}$ is an importance rating of the degree of water permeability of the soil; and

$y_{5}$ is an importance rating of the purpose of the reservoir.

The weight coefficients for Eqs. (5) and (6) are taken from Table S5.

The vulnerability index is calculated by Eqs. (5) or (6). It corresponds to the degree of environmental vulnerability from a chemically hazardous site for the $i$-th land plot (soil), soil or aquatic medium (Table 4).

Table 4. Scale for assessing the degree of environmental vulnerability

\begin{tabular}{|c|c|}
\hline $\begin{array}{l}\text { Intervals of the index of } \\
\text { environmental vulnerability } \\
\text { from a chemically hazardous } \\
\text { site for the i-th land plot (soil), } \\
\text { site or aquatic medium }\end{array}$ & $\begin{array}{l}\text { Degree of environmental } \\
\text { vulnerability from a chemically } \\
\text { hazardous site for the i-th land } \\
\text { plot (soil), site or aquatic } \\
\text { medium }\end{array}$ \\
\hline$E V I_{i}=0$ & Absents \\
\hline $0<E V I_{i} \leq 2$ & Low \\
\hline $2<E V I_{i} \leq 4$ & Medium \\
\hline $4<E V I_{i} \leq 6$ & Significant \\
\hline $6<E V I_{i}$ & High \\
\hline
\end{tabular}
from a chemically hazardous site

\subsection{Determining the Risk Level of Environmental Consequences as a Result of Emergencies at Chemically Hazardous Sites}

The risk level of environmental consequences resulting from emergencies at chemically hazardous sites is determined by various combinations of risk components: an environmental hazard index and environmental vulnerability index determined for the most pessimistic for environment scenario of an accident at a chemically hazardous site.

The matrix, presented in Table 5, is used for the convenience of conducting the risk assessment procedure. Five risk levels are established and color-coded: very low; minor; moderate; high; and critical.

The calculated indexes of environmental hazard and vulnerability and the data in Table 5 determine the risk level of environmental consequences as a result of emergencies at chemically hazardous sites for the $i$-th land plot (soil), site, or water mediums. As the general risk level of accidents, the highest level of all received for each type of accident is selected.

This creates the opportunity to make a map with different values of the risk levels of environmental consequences as a result of emergencies at chemically hazardous sites after calculating for many i-th land plots (soil), sites or water medium around the enterprise. Further, it can be combined with a topographic map of the area.

One of the main functions of the Information and Analytical System for Assessing the Environmental Consequences of Man-made Emergencies is to create a Map of Environmental Risk Zoning. This map allows the specialists to identify the most vulnerable places around a chemically hazardous site, develop a strategy aimed at reducing to an acceptable level by reducing risk sources and optimizing the location of hazard sources and the possibility of reducing the impact of criteria parameters on the risk level of environmental consequences as a result of emergencies at chemically hazardous sites.

\section{Results and Discussion}

The guidelines have a unified structure that can be used to determine and assess the environmental risk from possible man-made emergencies and analyze the state of environmental safety and the environmental impact of the activities of enterprises where various

Table 5. Matrix for assessing the level of risk of environmental consequences as a result of emergencies at chemically hazardous sites for the

\begin{tabular}{|c|c|c|c|c|c|}
\hline \multirow{2}{*}{ Environmental hazard index } & \multicolumn{5}{|c|}{ Environmental vulnerability index } \\
\hline & $\mathbf{0}$ & from 0 to 2 & from 2 to 4 & from 4 to 6 & more than 6 \\
\hline 0 & absents & absents & absents & absents & absents \\
\hline less than 2 & absents & very low & minor & moderate & high \\
\hline from 2 to 3 & absents & minor & moderate & high & high \\
\hline from 3 to 4 & absents & moderate & high & high & critical \\
\hline from 4 to 5 & absents & high & high & critical & critical \\
\hline
\end{tabular}
i-th land plot (soil), site or aquatic medium 
hazardous chemicals are stored and used in technological operations.

Developed guidelines have a systematic approach to assessing environmental risks. With this approach, the choice of risk assessment methods is also justified, which allows specialists, if necessary, the use of more targeted methods for the analysis, assessment, and processing of risks for definite technological processes.

It is necessary to have criteria that are the basis for judging the degree of manufacturing safety, for an objective assessment of the level of environmental hazard of an enterprise. They should take into account all types of activities of organizations operating hazardous chemical sites.

The most difficult and time-consuming task is to compile a unified analytical database containing a list of descriptors and their possible values provided by experts. The difficulty is that it is necessary to form a representative data array of grouped sets of criteria parameters describing each hazardous chemical and its participation in the technological process of an enterprise.

The presented methodology was tested at several enterprises in the central region of the Republic of Kazakhstan, in the territory of which there are chemically hazardous sites (Kazakhmys Smelting LLP, Arcelor Mittal Temirtau JSC, etc.).

As a test case of the presented methodology, let us consider an assessment of the risk level of environmental consequences of emergencies due to an accident at Kazakhmys Smelting LLP, Zhezkazgan.

\subsection{Testing the Methodology for Assessing Environmental Risk from Man-made Emergencies at Chemically Hazardous Sites}

Kazakhmys Smelting LLP, an important company in the life of the town of Zhezkazgan, has been chosen to assess the severity of environmental consequences from potential emergencies at CHS.

The main activity of Kazakhmys Corporation LLP is ore refining, the production of non-ferrous metals. We have studied the technological processes at a chemically hazardous site of this enterprise in Zhezkazgan town, such as the sulfuric acid production workshop.

As an example, we consider the results of a research for the (i-th) object (land plot) located 150 meters from the manufacturing sites where hazardous chemicals are used in technological processes. Using the Google Earth Pro application, we studied the topography of the area where the chemically hazardous site of this enterprise is located and the land plot ( $i$-th site) is, taken as an example. Fig. S1 demonstrates a topographic map of the area around the object of the study. These are the tanks for the storage of sulfuric acid, located on the territory of this workshop.

The research team collected the necessary information by filling out questionnaires to determine hazard and vulnerability indexes at the enterprise. After that, the questionnaires were sent to the relevant technical services of Kazakhmys Smelting LLP to assess environmental risk.

There are two hazardous chemicals in circulation in the workshop for the production of sulfuric acid. These are sulfuric acid and sulfur dioxide. There are seven tanks for storing sulfuric acid near the workshop building, four of them with a volume of $600 \mathrm{~m}^{3}$ and three with a volume of $2,100 \mathrm{~m}^{3}$ of each one. About half of these tanks are usually left empty. Sulfurous anhydride is a by-product of metallurgical manufacture and is delivered to the workshop from other workshops through a pipeline system, where it is immediately included in the sulfuric acid synthesis cycle. In this enterprise sulfur dioxide obtained as a result of ore roasting and transported through the pipeline has a relatively low concentration (not more than 5\%), and, in the case of a leak, is not able to harm the environment.

The wear coefficient of fixed assets used in the technological process of sulfuric acid does not exceed 0.01 , while the replacement coefficient of fixed assets is more than 0.95.

The total length of pipelines for sulfuric acid is about $5 \mathrm{~km}$. Sulfuric acid is pumped for irrigation through 3 branches of the pipeline and is carried out with a total of 40 pumps arranged in a series. 16 of them have a capacity of $315 \mathrm{~m}^{3} / \mathrm{h}$, and 24 pumps have a capacity of $280 \mathrm{~m}^{3} / \mathrm{h}$. The sulfuric acid circulation system is equipped with automatic shut-off valves directly in the piping. This CHS has the necessary tools to localize chemical accidents associated with the leakage of sulfuric acid. Trays are installed; there is a pit, and the appropriate reagents for neutralization are present.

All personnel of this CHS regularly and punctually undergo training in the rules of safe operation of technological equipment, and the appropriate actions in case of a chemical accident. The technological process at the enterprise is carried out around the clock. The mode of work and the rest of operators and technical personnel is two shifts lasting 12 hours each. Revision of instructions aimed at the safe operation of the CHS is carried out under current regulations. All necessary documentation is present in full. The technological process is equipped with automatic control and monitoring. The quality of maintenance and repair is good.

The questionnaire results of the personnel of the enterprise responsible for the operation of the technological equipment, its safe operation, the parameters for the equipment used at the sulfuric acid manufacture site, as well as the physicochemical, toxicological and other properties of the circulating hazardous chemicals are presented in tables S11-13 for sulfuric acid.

A cluster of criteria parameters has been formed to assess the index of environmental vulnerability from hazardous chemicals available on the workshop for the production of sulfuric acid of Kazakhmys Smelting LLP (Table S14).

Using the Google Earth Pro application, the distance from the sulfuric acid production workshop of Kazakhmys Smelting LLP to the considered land (i-th) site was determined to calculate the vulnerability index. The relief in this section was studied. Information on the soil filtration coefficient was obtained from the reports of experts from the state geological organization Kaznedra, who conducted geological surveys in this region.

The environmental hazard index from sulfuric acid circulating in the sulfuric acid production workshop of Kazakhmys Smelting LLP which is located at a distance of $150 \mathrm{~m}$ from the researched land (i-th) site is based on the data presented in Tables S11-13. It is determined by Eq. (3) as:

$$
\begin{gathered}
E H I_{i}=(0.05 \cdot(2+5+3)+0.04 \cdot 1+0.06 \cdot(1+4)+ \\
0.12 \cdot 2+0.17 \cdot 5)+(0.037 \cdot 5+0.035 \cdot 1+0.025 \cdot 1+ \\
0.03 \cdot(4+1)+0.033 \cdot 5+0.04 \cdot 4)+(0.07 \cdot 1+0.1 \cdot 1)=2.82
\end{gathered}
$$


Table 6. Risk Level of Environmental Consequences from an Emergency in the Sulfuric Acid Production Workshop for i-th Sites of the Territory around the Kazakhmys Smelting LLP, Zhezkazgan City

\begin{tabular}{|c|c|c|c|c|c|c|c|c|c|c|c|}
\hline $\mathbf{Y}, \mathbf{m}, \mathbf{m}$ & -500 & -400 & -300 & -200 & -100 & $\mathbf{0}$ & 100 & 200 & 300 & 400 & 500 \\
\hline-500 & Absents & Absents & Absents & Absents & Absents & Absents & Absents & Absents & Absents & Absents & Absents \\
\hline-400 & Absents & Absents & Absents & Absents & Absents & Absents & Absents & Absents & Absents & Absents & Absents \\
\hline-300 & Absents & Absents & Absents & Absents & Absents & Absents & Absents & Absents & Absents & Absents & Absents \\
\hline-200 & Absents & Absents & Absents & Absents & Absents & Absents & Absents & Absents & Absents & Absents & Absents \\
\hline-100 & Absents & Absents & Absents & Absents & Absents & Absents & Absents & Absents & Absents & Absents & Absents \\
\hline 0 & Absents & Absents & Absents & Moderate & Moderate & High & Absents & Absents & Absents & Absents & Absents \\
\hline 100 & Absents & Absents & Absents & Absents & Moderate & Moderate & Moderate & Absents & Absents & Absents & Absents \\
\hline 200 & Absents & Absents & Absents & Absents & Moderate & Moderate & Minor & Absents & Absents & Absents & Absents \\
\hline 300 & Absents & Absents & Absents & Absents & Absents & Absents & Absents & Absents & Absents & Absents & Absents \\
\hline 400 & Absents & Absents & Absents & Absents & Absents & Absents & Absents & Absents & Absents & Absents & Absents \\
\hline 500 & Absents & Absents & Absents & Absents & Absents & Absents & Absents & Absents & Absents & Absents & Absents \\
\hline
\end{tabular}

The calculated value of the environmental hazard index of a chemically hazardous site meets the Moderate degree of environmental hazard of a chemically hazardous site (Table 3) from sulfuric acid of the sulfuric acid production workshop of Kazakhmys Smelting LLP.

The index of environmental vulnerability from a chemically hazardous site for the land ( $i$-th) site, according to the data presented in table S14, is determined as:

The index of environmental vulnerability for land (i-th) site located 150 meters from the manufacturing site, according to the data presented in table S14, is determined by Eq. (5) as:

$$
E V I_{i}=0.4 \cdot 4+0.25 \cdot 1+0.15 \cdot 6+0.2 \cdot 1=2.95
$$

According to Table 4, the calculated vulnerability index corresponds to the Medium degree of environmental vulnerability from the chemically hazardous site of the Kazakhmys Smelting LLP sulfuric acid production workshop for the land (i-th) site.

The risk level of environmental consequences as a result of possible emergencies at a chemically hazardous site is assessed by the calculated indexes of environmental hazard and vulnerability. The maximum level of risk of environmental consequences, which is the final one for the sulfuric acid production workshop, is selected according to two calculated values. The risk level of environmental consequences for the land (i-th) site from the chemically hazardous site of the sulfuric acid production workshop of Kazakhmys Smelting LLP is Moderate.

A similar calculation can be made for other $i$-th sites located around the Kazakhmys Smelting LLP enterprise.

Table 6 demonstrates the distribution of the risk level of environmental consequences from an emergency in the sulfuric acid production workshop for $i$-th sites of the territory around the Kazakhmys Smelting LLP, Zhezkazgan city. The center with coordinates (0; 0 ) matches to the location of the workshop for the production of sulfuric acid of the Kazakhmys Smelting LLP. The coordinate system of this table is oriented relative to parts of the world. North-South corresponds to the ordinate Y (column), West-East, respectively, corresponds to the abscissa X (line). Cell sizes may vary depending on the required detailing of the examined area.

Thus, the risk assessment for the territory around the enterprise, along with the measures taken at the enterprise focused on the safe functioning of the equipment, showed the high efficiency of measures aimed at reducing environmental vulnerability. Prioritization of such events at the design stage of the CHS will significantly reduce the risk of chemical contamination of the environment.

\section{Conclusions}

The authors of this paper carried out the research, the result of which was the writing of Methodology for Environmental Risk Assessment from Emergencies at Chemically Hazardous Sites.

Researchers first determined the criteria and developed a methodological approach to forecasting the environmental consequences of emergencies at chemically hazardous sites. Unlike most of the existing methods that determine the risk of exposure to hazardous chemicals in the environment, the methodology proposed not only criteria related to the physicochemical and ecotoxicological properties of hazardous chemicals but also included criteria that characterize the safety of technological processes on CHS, actions of CHS personnel, and environmental vulnerability.

As a result of the expert study, questionnaires were developed that made it possible to establish the whole range of criteria parameters that determine the likelihood and severity of the environmental consequences of chemical accidents. As a result of the study, weightings for each of the criteria parameters were determined. The obtained results formed the basis of the methodological approach for calculating the environmental risk from emergencies at chemically hazardous sites.

The conducted research allowed the authors to collect data on the effectiveness of industrial and environmental safety measures at these enterprises at the main chemically hazardous sites in Central Kazakhstan. The enterprises of different industries with different 
technological processes were investigated. Testing the created methodology allowed the reliable determination of the weighting of the criteria parameters for forecasting the environmental consequences of emergencies at chemically hazardous sites.

As an example, the article presents the risk level assessment of the environmental consequences as a result of possible emergencies at the chemically hazardous site of Kazakhmys Smelting LLP in Zhezkazgan. The study was focused on a land plot. An analysis of the descriptor's values allows managers of chemically hazardous sites and employees of regulatory organizations to develop some organizational and technical measures aimed at reducing environmental hazards and the vulnerability of the environment and, accordingly, the level of risk of environmental consequences in general.

The developed methodology is the basis of the Information and Analytical System for Assessing the Environmental Consequences of Man-made Emergencies, which will facilitate the implementation of a set of measures aimed at predicting and reducing the impact of damaging factors of the emergency on the environment.

The introduction of information and analytical system for assessing the hazard level will allow local executive bodies to:

- objectively and expeditiously analyze the state of environmental safety at industrial sites;

- make justified management decisions aimed to reduce the risk to the environment around chemically hazardous sites; and

- monitor the effectiveness of applied measures.

All these measures allow a comprehensive approach to solving the problem of increasing the level of environmental safety at industrial sites.

Currently, the authors develop an information and analytical system in terms of creating the basics of developing information maps of regional environmental risk fields taking into account seasonal climatic conditions. This will allow users a more accurate adjustment of the structure of the risk source while, strengthening the process of management and control of environmental risk.

\section{Acknowledgment}

This work was supported by the Ministry of Education and Science of the Republic of Kazakhstan under Grant AP 05133965. The authors would like to thank Ben Taylor, EFL Specialist, Nur-Sultan, Kazakhstan, and Dr. Margaret Mary Nelson, Director of The Ethical Research Network Limited, London, for their assistance with the preparation of this article. The authors would like to thank the editors and anonymous reviewers for their insightful comments and suggestions.

\section{Author Contributions}

P.Y. (Professor) conducted research and wrote the manuscript. S.K. (Associate Professor) conducted research, wrote, and revised the manuscript. N.Y. (Lecturer) conducted research and wrote the manuscript.

\section{References}

1. Aven T. Risk assessment and risk management: Review of recent advances on their foundation. Eur. J. Oper. Res. 2016;253:1-13.

2. Khan F, Rathnayaka ., Ahmed S. Methods and models in process safety and risk management: Past, present and future. Process. Saf. Environ. Prot. 2015;98:116-147.

3. Republic of Kazakhstan. The Environmental Code of the Republic of Kazakhstan. [Internet]. 2007. [cited 11 August 2020]. Available from: https://online.zakon.kz/document/?doc_id= 30085593

4. Russian Research Institute for Issues of Civil Defense and Emergency Management. International Standard 22.0.05-97 Safety in emergencies. Technogenic emergencies. Terms and definitions. Moscow: Publishing House of Standards PPC; 2000.

5. BBC. Mexico's Sonora state reports new mine spill. [Internet]. London: BBC; 2014 [cited 1 July, 2020]. Available from: https://www.bbc.com/news/world-latin-america-29306026

6. Mexico News Daily. Buenavista copper mine fined 23mn. Mexico: Mexico News Daily; 2015 [cited 1 July, 2020]. Available from: https://mexiconewsdaily.com/news/buenavista-coppermine-fined-23mn/

7. Lee-Steere Ch. Environmental risk assessment guidance manual for industrial chemicals. Canberra: Environment Protection and Heritage Council; 2009. p. 109.

8. Häkkinen J, Kiiski A, Malk V, Myyrä M, Penttinen OP. Preparedness for chemical transport accident in Kymenlaakso, Evaluation of environmental risks and suitable remediation methods. Final report of the ChemRisk project. Helsinki: University of Helsinki; 2010. p. 140.

9. Häkkinen J, Malk V, Posti A, Penttinen OP, Mäkelä R, Kiiski A. Environmental risk assessment of the most commonly transported chemicals: case study of Finnish coastal areas. WMU J. Marit. Affairs 2013;12:147-160.

10. European Chemicals Agency. Guidance on information requirements and chemical safety assessment. Chapter R.16: Environmental exposure assessment. Helsinki: European Chemicals Agency; 2016. p. 178.

11. U. S. Environmental Protection Agency. Sustainable futures / P2 Framework manual 2012 EPA-748-B12-001. [Internet]. Washington: U. S. Environmental Protection Agency; 2012 [cited 12 November, 2019]. Available from: https://www.epa. gov/sites/production/files/2015-05/documents/05.pdf

12. Andersson AS. Development of an environment-accident index: a planning tool to protect the environment in case of a chemical accident [dissertation] Umea: University of Umea; 2004.

13. Shao Ch, Yang J, Tian X, Ju M, Huang L. Integrated environmental risk assessment and whole-process management system in chemical industry parks. Int. J. Environ. Res. Public Health 2013;10:1609-1630.

14. Ahmed I, Nazzal Y, Zaidi FK, Al-Arifi SN, Ghrefat H, Naeem M. Hydrogeological vulnerability and pollution risk mapping of the saq and overlying aquifers using the DRASTIC model and GIS techniques. NW Saudi Arabia. Environ. Earth Sci. 2015;74:1303-1318.

15. Ghazavi R, Ebrahimi Z. Assessing groundwater vulnerability to contamination in an arid environment using DRASTIC and 
GOD models. Int. J. Environ. Sci. Technol. 2015;12:2909-2918.

16. Wallace J, Payne N, Emerson RL. Sensitivity and vulnerability of the aquifers and springs in the Uinta Basin, Utah, to potential contamination associated with energy resource development. Geology of Utah's Uinta Basin and Uinta Mountains: Utah Geological Association Publication 2015;44:189-206.

17. Sharadqah S. Contamination risk evaluation of groundwater in the Canton of Portoviejo-Ecuador, using susceptibility index and two intrinsic vulnerability models. Amer. J. Environ. Sci. 2017;13:65-76.

18. Gesim NA, Okazaki T. Assessment of groundwater vulnerability to pollution using DRASTIC model and fuzzy logic in Herat city, Afghanistan. Int. J. Adv. Comp. Sci. App. 2018;9:181-189.

19. Kidam K, Hurme M, Hassim MH. Technical analysis of accident in chemical process industry and lessons learnt. Chem. Eng. Transactions 2010;19:451-456.

20. Cekirge HM. Environmental risk assessment methodology (ERAM) for oil pollution. Int. J. Environ. Monit. Anal. 2015;3:91-110.

21. Environment Directorate of the Organization for Economic Cooperation and Development. No. 29. Ageing of Hazardous Installations. Paris: OECD Environment, Health and Safety Publications Series on Chemical Accidents; 2017. p. 34.

22. Mackay D. Multimedia environmental models. Chelsea, Mich.: Lewis Publishers; 1991. p. 257.

23. Ziegler A. Chemical risk assessment - A tool for disaster prevention. J. Hazard. Mater. 1992;31:233-239.

24. Kudryavtsev SS, Yemelin PV, Yemelina NK. The development of a risk management system in the field of industrial safety in the Republic of Kazakhstan. Saf. Health Work 2018;9:30-41.

25. Yemelin PV, Kudryavtsev SS, Yemelina NK. Information and analytical system for hazard level assessment and forecasting risk of emergencies. Acta Polytech. 2019;59:182-191.

26. Andersson AS, Stjernström O, Fängmark I. Use of questionnaires and an expert panel to judge the environmental consequences of chemical spills for the development of an environ ment-accident index. J. Environ. Manage. 2005;75:247-261.

27. Andersson AS, Tysklind M, Fängmark I. A method to relate chemical accident properties and expert judgements in order to derive useful information for the development of Environment-Accident Index. J. Hazard. Mater. 2007;147:524-533.

28. Bodrikov OV, Yelokhin AP, Ryazantsev BV, Ryzhikov VS. Digest of methodologies for forecasting possible accidents, cata strophes, natural disasters in RFEM., Book 2. Moscow: Ministry of Russian Federation on Civil Defence, Emergencies, and the Liquidation of Consequences of Emergencies; 1994. p. 23.

29. Federal Mining and Technical Supervision of Russia. Methodological guidelines on the classification of accidents and incidents at hazardous industrial sites of chemical, pet rochemical and petroprocessing industry. $\mathrm{MD}$ 09-398-01. Moscow: Scientific and Technical Center for Industrial Safety Research CJSC; 2010; p. 180.

30. Gyenes Z, Wood M-H, Struckl M. Handbook of scenarios for assessing major chemical accident risks. Luxembourg: Publications Office of the European Union; 2017. p. 115.

31. European Parliament and Council. Seveso 2012/18/EU, Directive 2012/18/EU on the control of major-accident hazards involving dangerous substances, amending and subsequently repealing Council Directive 96/82/EC. [Internet]. 2012. [cited 11 December 2019]. Available from: https://eur-lex.europa.eu/legal-content/EN/TXT/HTML/?uri=CELEX: 32012L0018\&from=EN\#d1e32-19-1

32. Industrial and Research Institute for Engineering Surveys in Construction. International Standard 25100-95 Soils. Classification. Moscow: Standartinform; 1995.

33. Kesoretskikh II, Zotov SI. Methodology of the vulnerability assessment of natural complexes to antropogenic impacts. Vestnik of Baltic Federal University Named after I. Kant 2012;1:51-57. 